\title{
Performance Analysis of Two-Stage Signal Processing Structure in Antenna Array
}

\author{
Mingyue Zhai ${ }^{1, a}$
}

\section{Keywords: Signal processing; Smart antenna; Two-stage}

\begin{abstract}
Computation afford of adaptive antenna technology is the key factor limited its applications. This paper presented a new two-stage structure to solve this problem. By dividing array antennas into two group, two-stage structure can reduce computation afford with the same system performance of one-stage structure as the traditional adaptive antenna technology does. Algorithm and criterion in each stage can be selected independently according to the applications situations. Theoretical analyses of system performance in two-stage and one-stage structures are presented, and computation simulations are performed using RLS algorithm. Due to the low-complexity and low-computation, more antennas can be used to improve performance. For diversity receiving, it can help to improve SNR and less computation afford is obtained with the same performance as the traditional processing structure. The presented two-stage structure can be generalized easily to multiple-stage structure.
\end{abstract}

\section{Introduction}

In wireless communications, how to remove the influence from co-channel interference (CCI), multi-address interference (MAI) and multi-path fading has become the key aspect when system performance is analyzed. Adaptive antenna technology employs digital signal processing technologies to improve SINR through adjusting beam direction of antenna array.

Because there are more users and real time need in adaptive antenna system, the ability of rapid computation is necessary for adaptive antenna system. In fact, digital processing technologies have been the key factor that limits the applications of adaptive antenna system. Until now, a lot of documents have adopted antenna diversity to solve the computation afford. After processed by antenna diversities, such as selection combining, equal-gain combing and maximal-ration combining, array signals can be processed with adaptive algorithms (for example MLSE and CMA).

\section{System Model}

The presented algorithm is shown in Fig.1. Digital signal processing can be performed in base band or mid-frequency band as usually. Two stage processing algorithm, however, is employing. Through A/D transformation, signals on the array elements are divided into two groups, with signals on each group being processed independently and being output. The output signals on each group of antennas are input to process in DSP, general CPU or ASIC/FPGA.

With processing signals into two-stage, we can select different algorithms, even different criterions, according to applications needs and signals properties and signals quality and convergence speed are to be improved. At the same time, due to dividing, the number of antennas in each group is less, and the computation afford will be reduced. If RLS algorithm is used, computation afford is proportionate to square of array elements' number. 


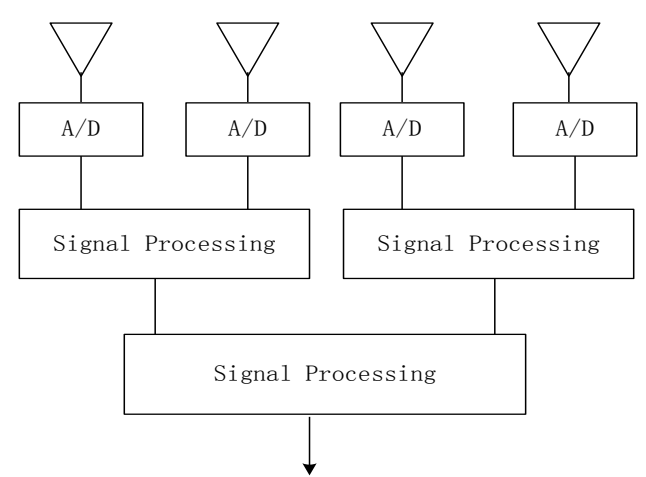

Figure. 1. The structure of the two-stage processing

\section{Performance Analysis}

We assume there are $N$ antennas and $M$ transmitting sources. The base band signal on the $n$th array element at $t=k T_{0}$ can be written as [2]:

$$
x_{n}(k)=\sum_{m=1}^{M}\left(h_{m}^{n}\right)^{T} a_{m}(k) e^{j k_{c}(n-1) l \sin \theta_{m}}+n_{n}(k)
$$

where $a_{m}(k)$ is the vector according to the $m$ th source, $h_{m}^{n}$ is the channel impulse response corresponding to the $m$ th source signal and the $n^{\text {th }}$ array element, $\theta_{m}$ is the DOA of the $m$ th source signal, $l$ is the inter-element distance, $k_{c}$ is the wave number, $n_{n}(k)$ is noise. If the adaptive algorithm is based on Weina solution and training sequence is used, the processed signal is the strongest one and the other signals are considered interference signals. When the number of interference signals is big enough, white Gauss noise is assumed in CDMA system. For simplicity, we assume only one signal and the powers between signal and noises are no different. Therefore, (1) can be simplified as:

$$
x_{n}(k)=h_{n} s(t) e^{i(n-1) \beta_{d}}+n_{n}(k)
$$

where $s(t)$ is the transmitted base band signal, $\beta_{d}$ is the phase difference between signals on different array elements.

The received signals on the antenna array can be written as:

$$
X(k)=S(k)+N(k)
$$

where,

$$
\begin{aligned}
& \begin{aligned}
S(k) & =\left[s(k) h_{1}, s(k) h_{2} e^{i \beta_{d}}, \cdots, s(k) h_{n} e^{i(N-1) \beta_{d}}\right]^{T} \\
& =s(k) \Gamma^{T}=\left[S_{1}(k), S_{2}(k), \cdots, S_{N}(k)\right]^{T} \\
N(k) & =\left[n_{1}(k), n_{2}(k), \cdots, n_{N}(k)\right]^{T}
\end{aligned}
\end{aligned}
$$

Due to stable signals and Gauss white noise, we can assume:

$$
E\left[S_{i}^{*} S_{i}\right]=P_{s}
$$

$E\left[n_{i}^{*} n_{i}\right]=P_{n}$

where, $*$ denotes conjugate.

From Wiena theorem, we can get [3]:

$$
w(k)=R_{x}(k)^{-1} R_{x X}(k)
$$


where,

$$
\begin{aligned}
& R_{x}(k)=E\left[X(k)^{*} X(k)^{T}\right] \\
& R_{x X}(k)=E\left[x(k) X(k)^{*}\right]
\end{aligned}
$$

To define:

$\rho=\frac{P_{s}}{P_{n}}$

Due to irrelevance among noises and between noises and the received signals, we can get:

$$
\begin{aligned}
& R_{x}(k)=P_{n}\left(I+\rho \Gamma^{H} \Gamma\right) \\
& R_{x X}(k)=P_{s} \Gamma^{H}
\end{aligned}
$$

where $\mathrm{H}$ denotes conjugate transpose. According to reverse matrix solution, we can get:

$$
\begin{aligned}
& R_{X}^{-1}(k)=P_{n l}^{-1}\left[I-\frac{\rho \Gamma^{H} \Gamma}{1+\rho M}\right] \\
& w(k)=\frac{\rho}{1+\rho M} \Gamma^{H}
\end{aligned}
$$

The output signals can be expressed as:

$$
\begin{aligned}
& \hat{s}(k)=w(k)^{H} X(k) \\
& =\frac{\rho N}{1+\rho N} s(k)+ \\
& \frac{\rho N}{1+\rho N}\left[n_{1}(k)+n_{2}(k) e^{j \beta_{d}}+\cdots+n_{N} e^{j(N-1) \beta_{d}}\right]
\end{aligned}
$$

This signal isn't the same as ideal signal, and it can be the signal affected by noised. Expression (16) include two items, with former being signal and latter being noise. We define the processed power of noise, which is different with [4]:

$$
P_{n}=E\left[\left|\hat{s}(k)-\frac{\rho N}{1+\rho N} s(k)\right|^{2}\right]
$$

The power of the interference and noise can be expressed as:

$$
P_{n}^{1}(k)=E\left[\left|\hat{s}(k)-\frac{\rho N}{1+\rho N} s(k)\right|^{2}\right]=\frac{\rho^{2} N}{(1+\rho N)^{2}} P_{n}
$$

SINR of the received signals after signal processed is:

$$
\rho_{1}=N \rho
$$

Now we analyses the system performance of two-stage signal processing structure.

Assuming there are $M N$ antennas and each group has $N$ antennas with $M$ groups in the first stage. In the second stage, $M$ output signals are processed, and the coefficients are:

$$
w_{1}(k)=\frac{\rho_{1}}{1+\rho_{1} M} \Gamma_{1}^{H}
$$

The optimal filtered signals are expressed as:

$$
\hat{s}(k)=w_{1}(k) \hat{s}(k)
$$


Because the input noises are irrelative in the second stage and noses and signals are irrelative, the processed power of interferences and noises is:

$$
P_{n}^{2}=\frac{\rho_{1}^{2} M}{\left(1+\rho_{1} M\right)^{2}} P_{n}^{1}=\frac{\rho_{1}^{2} M}{\left(1+\rho_{1} M\right)^{2}} \frac{\rho^{2} N}{(1+\rho N)^{2}} P_{n}
$$

The processed power of the signals is:

$$
P_{\hat{s}}=\frac{\rho_{1}^{2} M^{2} \rho^{2} N^{2}}{\left(1+\rho_{1} M\right)^{2}(1+\rho N)^{2}} P_{s}
$$

It is easily noted that SNR is $M N \rho$ after processed by two- stage processing structure. If expression (19) is used directly, the same result is also obtained. It should be noted that SNR is also $M N \rho$ if no two-stage structure is used. Therefore, the system performance between two-stage and one-stage has no theoretical difference.

Form the point of computation afford, two-stage structure has low-computation. This point can be explained with RLS as the example. In general, the computation complex is proportionate with the quantity of $(M N)^{2}$; however, the computation complex is relative to the quantity of $\left(M \times N^{2}+M^{2}\right)$ in two-stage structure. For example, if we use 16 array elements, the computation afford of two-stage structure is $\frac{5}{16}$ as that of one-stage structure.

\section{Simulations}

\begin{tabular}{|c|c|}
\hline $\begin{array}{l}\text { Generation of } \\
\text { signals, inte }\end{array}$ & $\begin{array}{l}\text { Output signals with RLS } \\
\text { algorithm under two- }\end{array}$ \\
\hline $\begin{array}{l}\text { rferences and } \\
\text { Gauss noise }\end{array}$ & $\begin{array}{l}\text { stage structure and } \\
\text { one--stage structure }\end{array}$ \\
\hline
\end{tabular}

Simulations are performed in MATLAB, and the flow structure is shown in Figure.2.
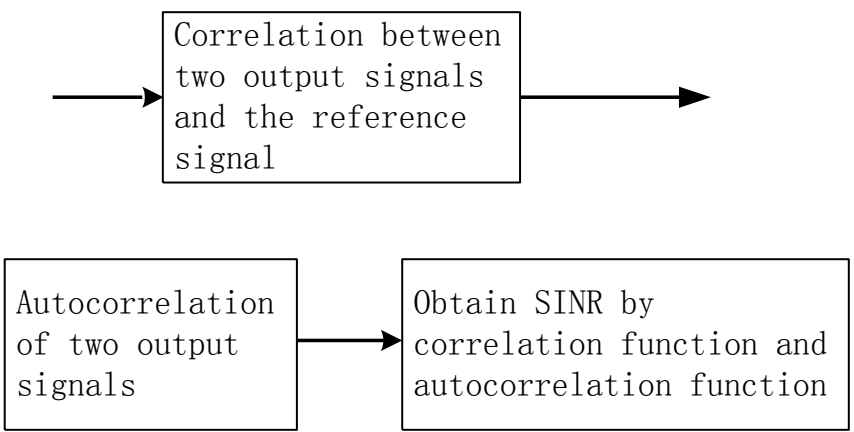

Figure. 2. The computer simulation flow diagram

The length of the reference sequence is 5000, and BPSK is used. Figure. 3 shows the results under array element's number ranging from 2 to 16 , and no interference signals are taken into account with noise power being $0 \mathrm{~dB}$ 


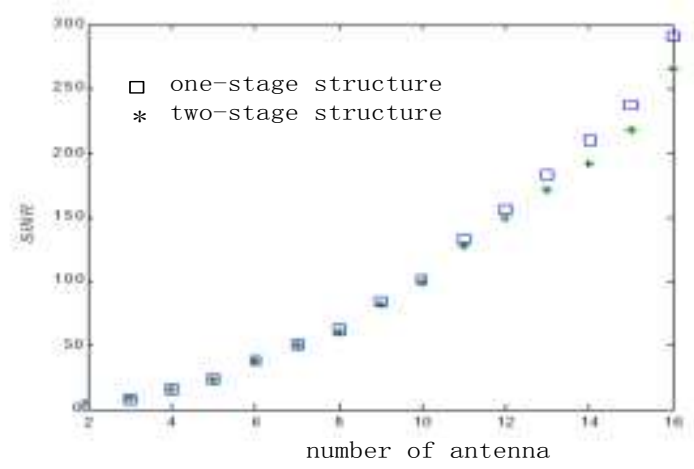

Figure. 3. The relation between SINR improved multiples and number of antenna

In Figure. 4, there are 25 array elements in one-stage structure and 5 antennas in the first stage for two-stage structure. The results are obtained under interference number arranging 1 to 40 . The amplitude of signal is 1 , while amplitude of each interference signal is $1 / 20$ and noise 1 .

From Figure. 3, Figure.4 and Figure.5, we can that system performance under one-stage structure and two-stage structure is similar.
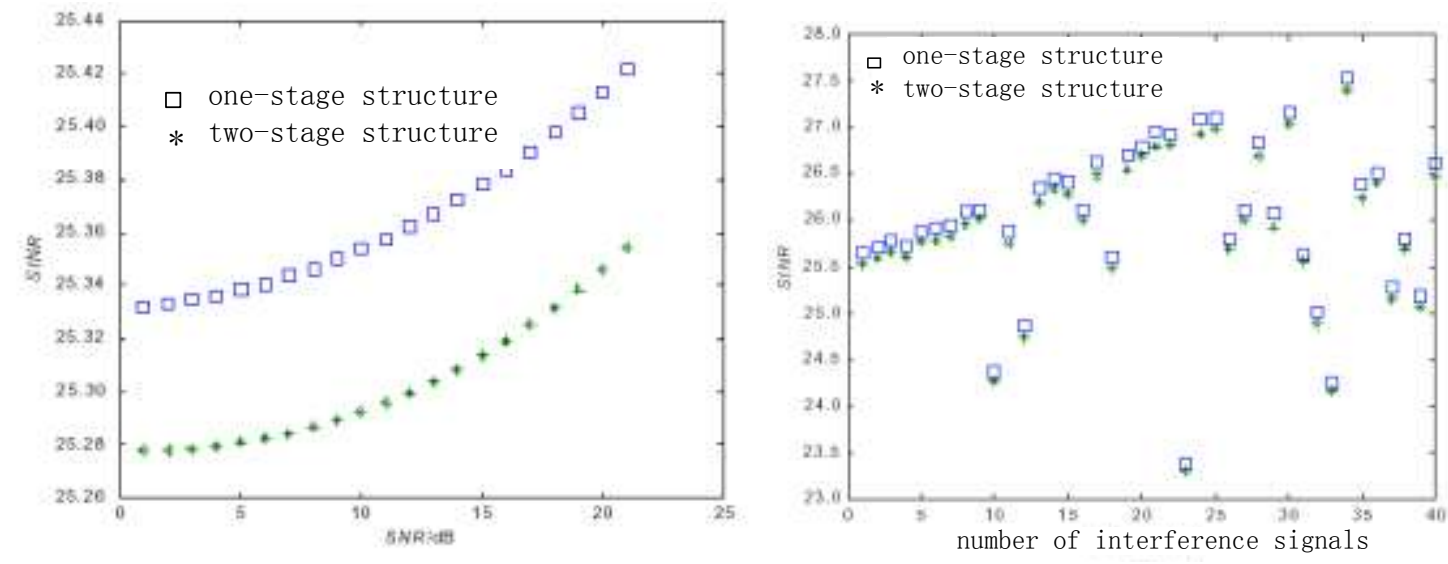

Figure. 4. Improved multiples of SINR under different input SNR Figure. 5. Improved multiples of SINR under different number of interference signals

\section{Conclusions}

In this paper, we presented a two-stage structure to process signals, and the performance analysis is also done. Due to the low-complexity and low-computation, more antennas can be used to improve performance. For diversity receiving, it can help to improve SNR and less computation afford is obtained with the same performance as the traditional processing structure. Owing to the characteristic of two-stage structure, algorithm and criterion can be selected according to the real applications situations. The presented two-stage structure can be generalized easily to multiple -stage structure

\section{References}

[1] Sayo H., Miyake M., Fujino T.. "Performance of diversity combing scheme using simplified weighting factor", IEICE Transactions on Communications, E80-B (8), pp.1160-1165, Aug. 1997.

[2] Ertel R B, et al, "Overview of spatial channel models for antenna array communications systems", IEEE Personal Communications, vol. 5(1), pp.10-22, Jan. 1998.

[3] Haykin S, Adaptive Filter Theory, Press of Electrics Industrial, Beijing, 1998. 
[4] Odile M. M., Neil J. B.. "Adaptive recovery of a chirped sinusoid in noise, part 1: performance of the RLS algorithm”, IEEE Transaction on Signal Processing, vol. 39 (3), pp.583-594, Mar. 1991.

[5] Leou M. L., Li H. J.. "A novel hybrid of adaptive array and equalizer for mobile communications", IEEE Transaction on Vehicular Technology, vol. 49 (1), pp.1-10, Jan. 2014 